\title{
ON AN ALGORITHM FOR THE PROBLEM OF TRACKING A TRAJECTORY OF A PARABOLIC EQUATION
}

\author{
MARINA BLIZORUKOVA ${ }^{a, b, *}$, VYACHESLAV MAKSIMOV ${ }^{a, b}$ \\ ${ }^{a}$ Institute of Mathematics and Mechanics \\ Ural Branch of the Russian Academy of Sciences, S. Kovalevskaya 16, Yekaterinburg 620990, Russia \\ e-mail: msbeimm.uran.ru \\ ${ }^{b}$ Graduate School of Economics and Management \\ Ural Federal University, Yekaterinburg 620002, Russia
}

\begin{abstract}
In this paper, we consider the problem of tracking a solution of a reference parabolic equation by a solution of another equation. A stable algorithm based on the extremal shift method is proposed for this problem. The algorithm is designed to work on a sufficiently large time interval where both equations operate.
\end{abstract}

Keywords: parabolic equation, tracking problem, unknown disturbance.

\section{Introduction}

In this paper, the problem of tracking of a trajectory a parabolic equation subject to an unknown disturbance is considered. Tracking control problems for distributed parameter systems has attracted considerable attention recently. They have been investigated, for example, by Grimble and Johnson (1988), Sontag (1990), Pandolfi and Priola (2005), or Prodan et al. (2013). Methods for solving similar problems are presented, in particular, within the theory of positional control (Krasovskii and Subbotin, 1988).

Here, we study the tracking problem on an infinite time interval. The framework considered has one peculiarity. It is assumed that the current states of a given control system and an etalon system (influenced by an uncontrollable disturbance) are observed with small errors. This assumption implies the impossibility to track precisely the trajectory of the etalon system by that of the given control system. Taking into account this aspect, we construct a feedback control algorithm stable with respect to informational noise and computational errors. This algorithm is based on the method of extremal shift from the theory of positional differential games. Prototypes of the tracking problem on an infinite time interval for dynamical systems described by ordinary

* Corresponding author differential equations have been considered by Maksimov (2011) or Kryazhimskiy and Maksimov (2011). In the present paper, in contrast to the works mentioned above, we investigate a distributed parameters system.

\section{Problem statement and the solution method}

In a Hilbert space $(X,|\cdot| X)$, we consider a parabolic equation of the form

$$
\begin{aligned}
\dot{x}(t)=A x(t)+ & B u(t)+f(t), \\
t & \in T=[0,+\infty), \quad x(0)=x_{0},
\end{aligned}
$$

where $A$ is the infinitesimal generator of a strongly continuous semigroup of bounded linear operators $\mathcal{X}(t): X \rightarrow X(t \in T), f(\cdot) \in L_{2}(T ; X)$ is a given disturbance, $B$ is a continuous linear operator $(B \in$ $\mathcal{L}(U ; X)), U$ is a Hilbert space with norm $|\cdot|_{U}$ and inner product $(\cdot, \cdot)_{U}$.

A weak solution of Eqn. (1) corresponding to a control $u(\cdot) \in L_{\infty}(T ; U)$ and an initial state $x(0)=x_{0}$ is a continuous function $x(t): T \rightarrow X$ defined by the formula

$$
x(t)=\mathcal{X}(t) x_{0}+\int_{0}^{t} \mathcal{X}(t-\tau)\{B u(\tau)+f(\tau)\} \mathrm{d} \tau .
$$


As is known, for any $x_{0} \in X$, and $u(\cdot) \in L_{\infty}(T ; U)$, there exists a unique weak solution $x\left(\cdot ; 0, x_{0}, u(\cdot)\right) \in C(T ; X)$ of Eqn. (1) of the form (2).

The problem under consideration may be formulated in the following way. Equation (1) is influenced by an unknown control $u(t) \in P$. Here, $P \subset U$ is a bounded closed set, some control resource (a set of instantaneous restrictions on the control). At discrete, frequent enough times $\tau_{i} \in T(i=1,2, \ldots)$, the phase state $x\left(\tau_{i}\right)$ of (1) is inaccurately measured. The results of these measurements, i.e., elements $\xi_{i}^{h} \in X$, satisfy the inequalities

$$
\left|\xi_{i}^{h}-x\left(\tau_{i}\right)\right|_{X} \leq \nu_{i}^{h},
$$

where $\nu_{i}^{h} \in(0,1)$ stands for a level of informational error at the moment $\tau_{i}$, and the number $h \in(0,1)$ characterizes the measurement accuracy. Along with Eqn. (1), there is an additional equation of the same form,

$$
\dot{y}(t)=A y(t)+B v(t)+f(t), \quad t \in T,
$$

with an initial state $y(0)=y_{0}$. In what follows, we call it the etalon equation. The solution $y(\cdot)$,

$$
y(t)=\mathcal{X}(t) y_{0}+\int_{0}^{t} \mathcal{X}(t-\tau)\{B v(\tau)+f(\tau)\} \mathrm{d} \tau,
$$

of this equation is generated by a control

$$
\begin{aligned}
& v(\cdot) \in P(\cdot)=\left\{v(\cdot) \in L_{2}(T ; U):\right. \\
& \qquad v(t) \in P \text { for a.a. } t \in T\} .
\end{aligned}
$$

The etalon control $v(\cdot)$ and the corresponding solution $y(\cdot)=y\left(\cdot ; 0, y_{0}, v(\cdot)\right)$ of Eqn. (4) are unknown. At discrete times $\tau_{i} \in T(i=1,2, \ldots)$, along with $x\left(\tau_{i}\right)$, the state $y\left(\tau_{i}\right)=y\left(\tau_{i} ; 0, y_{0}, v(\cdot)\right)$ of Eqn. (4) is inaccurately measured. The results of measurements are elements $\psi_{i}^{h} \in X, i=1,2, \ldots$ satisfying the inequalities

$$
\left|y\left(\tau_{i}\right)-\psi_{i}^{h}\right|_{X} \leq \nu_{i}^{h} .
$$

We assume that the initial states $x_{0}, y_{0}$ satisfy the relation

$$
\left|y_{0}-x_{0}\right|_{X} \leq h .
$$

The problem considered in the paper consists in the following: It is necessary to design a real-time algorithm that, using the results of measurements $\xi_{i}^{h}$ and $\psi_{i}^{h}$, forms a control $u=u^{h}(\cdot)$ allowing us to track the solution $y(\cdot)$ of Eqn. (4) by the solution $x(\cdot)$ of Eqn. (1) in such a way that the deviation of $y(\cdot)$ from $x(\cdot)$ (on the interval $T$ ) is arbitrarily small provided the measurement accuracy $h$ is small enough. We assume that the algorithm should be stable with respect to informational noise and computational errors. In what follows, the solution of Eqn. (1) generated by the control $u=u^{h}(\cdot)$ is denoted by the $\operatorname{symbol} x^{h}(\cdot)=x\left(\cdot ; 0, x_{0}, u^{h}(\cdot)\right)$.
For a bounded interval, this problem can be solved using the constructions from the works of Osipov (2009), Maksimov (2002; 2012; 2013), Blizorukova et al. (2001), or Kapustyan and Maksimov (2014). Note that the algorithms proposed in these works are oriented to a finite time interval. As its length is increased, the computational and measurement errors are accumulated. Algorithms that are free from this disadvantage were considered by Maksimov (2011) as well as Kryazhimskiy and Maksimov (2011) for systems of ordinary differential equations, and by Maksimov (2014) for a parabolic equation. A functional-analytic representation of the solution was used in the latter case. Here, we apply the semigroup representation of solutions.

Let for every $h \in(0,1)$ a family $\Delta_{h}$ of partitions of the half-axe $[0,+\infty)$ by times $\tau_{h, i}$ be fixed:

$$
\begin{gathered}
\Delta_{h}=\left\{\tau_{h, i}\right\}_{i=0}^{\infty}, \quad \tau_{h, 0}=0, \quad \tau_{h, i+1}=\tau_{h, i}+\delta_{i}(h), \\
\delta_{i}(h) \in(0,1), \quad \sum_{i=0}^{+\infty} \delta_{i}(h)=+\infty \quad \forall h \in(0,1) .
\end{gathered}
$$

Here, $\delta_{i}(h)$ is the diameter of the $i$-th step of the family $\Delta_{h}$, i.e., the distance between the times $\tau_{h, i+1}$ and $\tau_{h, i}$. Note that the latter equality in (7) shows that the moments $\tau_{h, i}$ cover the whole interval $T$.

In what follows, by $\Xi(x(\cdot), h)$ we denote the set of all piecewise constant functions $\xi^{h}(\cdot):[0,+\infty) \mapsto X$, $\xi^{h}(t)=\xi_{i}^{h}$ for $t \in\left[\tau_{h, i}, \tau_{h, i+1}\right), i \geq 0, \xi_{0}^{h}=x_{0}$, satisfying the inequalities (3). In turn, by $\Xi(y(\cdot), h)$ we denote the set of all piecewise constant functions $\psi^{h}(\cdot)$ : $[0,+\infty) \mapsto X, \psi^{h}(t)=\psi_{i}^{h}$ for $t \in\left[\tau_{h, i}, \tau_{h, i+1}\right), i \geq 0$, $\psi_{0}^{h}=y_{0}$, satisfying the inequalities (5).

Below, we assume that the following condition is fulfilled.

Condition 1. The norm $|\cdot|{ }_{2}$ (equivalent to the norm $|\cdot|_{X}$ ) is introduced in the space $X$ :

$$
\begin{gathered}
c_{1}|\cdot|_{2} \leq|\cdot| X \leq c_{2}|\cdot|_{2}, \\
c_{1}, c_{2}=\text { const } \in(0,+\infty), c_{1}<c_{2} ;
\end{gathered}
$$

with this norm, the semigroup $\mathcal{X}(t)$ is $\omega$-dissipative, i.e.,

$$
|\mathcal{X}(t) x|_{2} \leq \exp (\omega t)|x|_{2} \quad \text { for any } \quad x \in X .
$$

The norm $|\cdot|_{2}$ is generated by some inner product $(\cdot, \cdot)_{2}$.

Below, as is often done, we identify the space $X$ with the dual space $X^{*}$.

As a criterium for the deviation of the solution $x^{h}(\cdot)$ of Eqn. (1) from the solution $y(\cdot)$ of Eqn. (2) on a bounded time interval $[0, \vartheta]$, we consider the value

$$
\begin{aligned}
& W\left(x^{h}(\cdot), y(\cdot) \mid \vartheta\right) \\
& =\max _{0 \leq \tau_{i} \leq \vartheta} \exp \left(-2 \omega \tau_{i}\right) \mid y\left(\tau_{i} ; 0, y_{0}, v(\cdot)\right) \\
& \quad-\left.x^{h}\left(\tau_{i} ; 0, x_{0}, u^{h}(\cdot)\right)\right|_{2} ^{2}, \quad \tau_{i}=\tau_{h, i} .
\end{aligned}
$$




\section{Solution algorithm}

Before describing an algorithm for solving the problem in question, we formulate two conditions.

Condition 2. The family of partitions $\Delta_{h}$ given by (7) and the values of measurement errors $\nu_{i}^{h}$ are such that the following relations are valid:

$$
\begin{gathered}
\nu_{0}^{h} \leq c_{*} h \quad\left(c_{*}=\text { const }>0\right), \\
\nu_{i}^{h} \in(0,1) \text { for all } i=0,1, \ldots \text { and all } h \in(0,1), \\
\sum_{i=0}^{+\infty} \delta_{i}(h) \nu_{i}^{h} \leq \varphi_{1}(h) \rightarrow 0+\quad \text { as } h \rightarrow 0+.
\end{gathered}
$$

Condition 3. The inequality

$$
\sum_{i=0}^{+\infty} \delta_{i}^{2}(h) \leq \varphi_{2}(h)
$$

holds as $h \rightarrow 0$.

Condition 2 substantially means that the noise signals implemented in the observation channel are subject to the constraints of smallness of their mean values over the whole time interval $T$.

Remark 1. Conditions 2 and 3 hold, for example, if

$$
\begin{gathered}
\delta_{i}(h)=\nu_{i}^{h}=\frac{d_{0} h}{(i+1)^{\mu}} \leq 1, \\
\mu \in(0.5 ; 1], \quad i=0,1, \ldots, \quad d_{0}=\text { const }>0 .
\end{gathered}
$$

In this case

$$
\varphi_{1}(h)=\varphi_{2}(h)=2 h^{2} d_{0}^{2} \sum_{i=1}^{\infty} i^{-2 \mu},
$$

and the inequalities (3) take the form

$$
\left|\xi_{i}^{h}-x\left(\tau_{i}\right)\right|_{X} \leq \frac{d_{0} h}{(i+1)^{\mu}}
$$

Before the algorithm starts, we fix a value $h \in(0,1)$, a family $\left\{\nu_{i}^{h}\right\}_{i=0}^{\infty}$, and a partition $\Delta_{h}$ (see (7)). The work of the algorithm is decomposed into identical steps. At the $i$-th step carried out on the time interval $\left[\tau_{i}, \tau_{i+1}\right)$, the following operations are fulfilled. First, at the time $t=\tau_{i}$, the phase states $x^{h}\left(\tau_{i}\right)$ and $y\left(\tau_{i}\right)$ of Eqns. (1) and (4) are measured; i.e., the elements $\xi_{i}^{h} \in X$ and $\psi_{i}^{h} \in X$ with the properties (3) and (5) are found. Then, a function $u^{h}(\cdot)$ (Lebesque measurable or piecewise continuous) satisfying the inequality

$$
\begin{aligned}
& \left(B^{*} \mathcal{X}^{*}\left(\tau_{i+1}-\tau\right) \tilde{s}_{i}, u^{h}(\tau)\right)_{2} \\
& \leq \inf \left\{\left(B^{*} \mathcal{X}^{*}\left(\tau_{i+1}-\tau\right) \tilde{s}_{i}, u\right)_{2}: u \in P\right\} \\
& \quad+d \nu_{i}^{h} \quad \text { for a.a. } \tau \in\left[\tau_{i}, \tau_{i+1}\right)
\end{aligned}
$$

is calculated. Here $d=$ const $>0$ is an a priori fixed constant and $B^{*}$ is the adjoint operator,

$$
\tilde{s}_{i}=\mathcal{X}\left(\tau_{i+1}-\tau_{i}\right) s_{i}, \quad s_{i}=\xi_{i}^{h}-\psi_{i}^{h}
$$

Then the control $u^{h}(t)$ is fed onto the input of Eqn. (1) during the interval $\left[\tau_{i}, \tau_{i+1}\right)$. Thereby, at the moment $\tau_{i}$, the program control $u^{h}(\tau), \tau \in\left[\tau_{i}, \tau_{i+1}\right)$, is calculated. Under the action of this control, the phase trajectory of Eqn. (11) passes from the state $x^{h}\left(\tau_{i}\right)$ to the state $x^{h}\left(\tau_{i+1}\right)=x\left(\tau_{i+1} ; \tau_{i}, x^{h}\left(\tau_{i}\right), u^{h}(\cdot)\right)$.

Theorem 1. Let Conditions 1-3 hold. Then

$$
W\left(x^{h}(\cdot), y(\cdot) \mid \vartheta\right) \leq \nu(h)
$$

for all $\vartheta \geq 0$, where $\nu(h)=b_{1}\left(h^{2}+\varphi_{1}(h)+\varphi_{2}(h)\right)$ and $b_{1}$ is a constant which is independent of $\vartheta$ and whose closed form can be written explicitly.

Proof. Let $\xi^{h}(\cdot) \in \Xi\left(x^{h}(\cdot), h\right), \psi^{h}(\cdot) \in \Xi(y(\cdot), h)$. Let us estimate the variation of the functional

$$
\varepsilon_{h}(t)=\exp (-2 \omega t)\left|x^{h}(t)-y(t)\right|_{2}^{2}
$$

on the time interval $T$. For $t \in \delta_{i}$, we have

$$
\begin{aligned}
& x^{h}(t)=\mathcal{X}\left(t-\tau_{i}\right) x^{h}\left(\tau_{i}\right)+\int_{\tau_{i}}^{t} \mathcal{X}(t-\tau)\left\{B u^{h}(\tau)+f(\tau)\right\} \mathrm{d} \tau, \\
& y(t)=\mathcal{X}\left(t-\tau_{i}\right) y\left(\tau_{i}\right)+\int_{\tau_{i}}^{t} \mathcal{X}(t-\tau)\{B v(\tau)+f(\tau)\} \mathrm{d} \tau,
\end{aligned}
$$

see (2). Thus, by virtue of (8), for all $i=0,1, \ldots$, the estimate

$$
\begin{aligned}
\varepsilon_{h}\left(\tau_{i+1}\right) & \\
= & \exp \left(-2 \omega \tau_{i+1}\right) \mid \mathcal{X}\left(\delta_{i}(h)\right)\left(x^{h}\left(\tau_{i}\right)-y\left(\tau_{i}\right)\right) \\
& +\left.\int_{\tau_{i}}^{\tau_{i+1}} B\left\{u^{h}(\tau)-v(\tau)\right\} \mathrm{d} \tau\right|_{2} ^{2} \\
= & \exp \left(-2 \omega \tau_{i+1}\right)\left\{\left|\mathcal{X}\left(\delta_{i}(h)\right)\left(x^{h}\left(\tau_{i}\right)-y\left(\tau_{i}\right)\right)\right|_{2}^{2}\right. \\
& +2\left(\mathcal{X}\left(\delta_{i}(h)\right)\left(x^{h}\left(\tau_{i}\right)-y\left(\tau_{i}\right)\right),\right. \\
& \left.\int_{\tau_{i}}^{\tau_{i+1}} B\left\{u^{h}(\tau)-v(\tau)\right\} \mathrm{d} \tau\right){ }_{2} \\
& \left.+\left|\int_{\tau_{i}}^{\tau_{i+1}} B\left\{u^{h}(\tau)-v(\tau)\right\} \mathrm{d} \tau\right|_{2}^{2}\right\} \\
\leq & \exp \left(-2 \omega \tau_{i+1}\right)\left|\mathcal{X}\left(\delta_{i}(h)\right)\left(x^{h}\left(\tau_{i}\right)-y\left(\tau_{i}\right)\right)\right|_{2}^{2} \\
& +\lambda_{i}+\mu_{i},
\end{aligned}
$$


is valid. Here

$$
\begin{gathered}
\lambda_{i}=2\left(S_{i}, \int_{\tau_{i}}^{\tau_{i+1}} \mathcal{X}\left(\tau_{i+1}-\tau\right) B\left\{u^{h}(\tau)-v(\tau)\right\} \mathrm{d} \tau\right)_{2}, \\
\mu_{i}=c_{1} \delta_{i}(h) \exp \left(-2 \omega \tau_{i+1}\right) \int_{\tau_{i}}^{\tau_{i+1}}\left|B\left\{v(\tau)-u^{h}(\tau)\right\}\right|_{2}^{2} \mathrm{~d} \tau, \\
\delta_{i}(h)=\tau_{i+1}-\tau_{i}, \quad \tau_{i}=\tau_{h, i}, \\
S_{i}=\exp \left(-2 \omega \tau_{i+1}\right) \mathcal{X}\left(\delta_{i}(h)\right)\left[x^{h}\left(\tau_{i}\right)-y\left(\tau_{i}\right)\right] .
\end{gathered}
$$

In addition, by taking into account the $\omega$-dissipativity of the semigroup $\mathcal{X}(t)$, for all $i$, we have

$$
\exp \left(-2 \omega \tau_{i+1}\right)\left|\mathcal{X}\left(\delta_{i}(h)\right)\left(x^{h}\left(\tau_{i}\right)-y\left(\tau_{i}\right)\right)\right|_{2}^{2} \leq \varepsilon\left(\tau_{i}\right) .
$$

It is easily seen that

$$
\mu_{i} \leq c_{2} \delta_{i}^{2}(h)
$$

In turn, by virtue of the inequalities

$$
\left|\tilde{s}_{i}-S_{i}\right|_{2} \leq c_{3} \nu_{i}^{h}, \quad i=0,1, \ldots,
$$

we get

$$
\begin{aligned}
\lambda_{i} \leq & 2 \exp \left(-2 \omega \tau_{i+1}\right) \\
& \times\left(\tilde{s}_{i}, \int_{\tau_{i}}^{\tau_{i+1}} \mathcal{X}\left(\tau_{i+1}-\tau\right) B\left\{u^{h}(\tau)-v(\tau)\right\} \mathrm{d} \tau\right)_{2} \\
& +c_{4} \nu_{i}^{h} \delta_{i}(h), \quad i=0,1, \ldots
\end{aligned}
$$

Taking into account the rule of forming the control $u^{h}(\cdot)$ (see (9)), we obtain

$$
\begin{aligned}
& \exp \left(-2 \omega \tau_{i+1}\right)\left(B^{*} \mathcal{X}^{*}\left(\tau_{i+1}-\tau\right) \tilde{s}_{i}, u^{h}(\tau)\right)_{2} \\
& \leq \inf \left\{\exp \left(-2 \omega \tau_{i+1}\right)\left(B^{*} \mathcal{X}^{*}\left(\tau_{i+1}-\tau\right) \tilde{s}_{i}, u\right)_{2}:\right. \\
& u \in P\}+d_{*} \nu_{i}^{h} \quad \text { for a.a. } \quad \tau \in\left[\tau_{i}, \tau_{i+1}\right),
\end{aligned}
$$

where $d_{*}>0$ is a constant whose closed form can be written explicitly. From (14) and the last inequality, we get

$$
\lambda_{i} \leq c_{5} \nu_{i}^{h} \delta_{i}(h) .
$$

Combining (12)-(15), we conclude the estimate

$$
\begin{aligned}
& \varepsilon_{h}\left(\tau_{i+1}\right) \\
& \quad \leq \varepsilon_{h}\left(\tau_{i}\right)+c_{2} \delta_{i}^{2}(h)+c_{5} \nu_{i}^{h} \delta_{i}(h) \\
& \quad \leq \varepsilon_{h}\left(\tau_{i}\right)+c_{6} \delta_{i}(h)\left(\nu_{i}^{h}+\delta_{i}(h)\right), \quad i=0,1, \ldots
\end{aligned}
$$

Thus, for all $i=0,1, \ldots$, we get

$$
\varepsilon_{h}\left(\tau_{i}\right) \leq \varepsilon_{h}(0)+c_{6} \sum_{i=0}^{\infty} \delta_{i}(h)\left\{\nu_{i}^{h}+\delta_{i}(h)\right\} .
$$

Therefore, by (6)-(11), we have

$$
\varepsilon_{h}(0) \leq c_{7} h^{2} .
$$

From (16), by taking into account Conditions 2 and 3 and the latter inequality, we obtain (for all $i=0,1, \ldots$ )

$$
\varepsilon_{h}\left(\tau_{i}\right) \leq b_{1}\left(h^{2}+\varphi_{1}(h)+\varphi_{2}(h)\right),
$$

which implies (10). The proof of the theorem is complete.

Let the set of instantaneous restrictions on the control have the following form:

$$
\begin{aligned}
P=\left\{u \in U: u=\sum_{j=1}^{m} \omega_{j} u_{j}, \quad \omega_{j} \in U\right. & \\
& \left.u_{j} \in R, u=\left\{u_{1}, \ldots, u_{m}\right) \in P_{1} \subset \mathbb{R}^{m}\right\},
\end{aligned}
$$

where $P_{1}$ is a given bounded and closed set. Let also the control $v(t)$ on the right-hand side of Eqn. (4) have the following structure:

$$
v(t)=\sum_{j=1}^{m} \omega_{j} v_{j}(t)
$$

In this case, it is natural to choose the control $u=$ $u^{h}(\cdot)$ on the right-hand side of Eqn. (1) of the same structure as the control $v(\cdot)$. Thus, the controls are finite vector functions with values in the $m$-dimensional Euclidean space. Set

$$
u^{h}(t)=\sum_{j=1}^{m} u_{j i}^{h} \omega_{j} \quad \text { for a. a. } t \in\left[\tau_{i}, \tau_{i+1}\right) .
$$

Here, the vector $u_{i}^{h}=\left\{u_{1 i}^{h}, \ldots, u_{m i}^{h}\right\} \in P_{1}$ satisfies the inequality

$$
\begin{gathered}
\sum_{j=1}^{m} u_{j i}^{h}\left(\mathcal{X}\left(\delta_{i}\right)\left[\xi_{i}^{h}-\psi_{i}^{h}\right], B \omega_{j}\right)_{2} \\
\leq \inf \left\{\sum_{j=1}^{m} v_{j}\left(\mathcal{X}\left(\delta_{i}\right)\left[\xi_{i}^{h}-\psi_{i}^{h}\right], B \omega_{j}\right)_{2}:\right. \\
\left.v=\left\{v_{1}, \ldots, v_{m}\right\} \in P_{1}\right\}+d \nu_{i}^{h},
\end{gathered}
$$

where, as above, $\delta_{i}=\delta_{i}(h)=\tau_{h, i+1}-\tau_{h, i}$.

Remark 2. As can be seen from the above rule of forming the control $u^{h}(\cdot)$ (see (17) and (18)), this control is a piecewise constant function with discontinuities at the nodes of the partition $\Delta_{h}$.

Let the solution of Eqn. (4) generated by the control $v(\cdot)$ remain in a bounded domain; i.e., let the following condition be fulfilled.

Condition 4. $\sup \left\{\left|y\left(t ; 0, y_{0}, v(\cdot)\right)\right|_{X}: t \in T\right\}<\infty$. 
Theorem 2. Assume that the conditions of Theorem 1 and Condition 4 are fulfilled. Let also

$$
\varphi_{3}(h)=\sum_{i=0}^{\infty} \delta_{i}(h) \mu\left(\delta_{i}(h)\right) \rightarrow 0 \quad \text { as } h \rightarrow+0,
$$

where

$$
\mu(\delta)=\sup _{t \in[0, \delta]}\left|\mathcal{X}(t) \sum_{j=1}^{m} B \omega_{j}-\sum_{j=1}^{m} B \omega_{j}\right|_{2} .
$$

Then the assertion of Theorem 1 holds if the control $u^{h}(\cdot)$ is calculated by formulas (17) and (18). In this case,

$$
\nu(h)=b_{2}\left(h^{2}+\varphi_{1}(h)+\varphi_{2}(h)+\varphi_{3}(h)\right) .
$$

Proof. Taking into account Condition 4 , we conclude that

$$
\begin{aligned}
& \int_{\tau_{i}}^{\tau_{i+1}}\left(\tilde{s}_{i}, \mathcal{X}\left(\tau_{i+1}-\tau\right) B u^{h}(\tau)\right)_{2} \mathrm{~d} \tau \\
& -\int_{\tau_{i}}^{\tau_{i+1}}\left(\tilde{s}_{i}, \mathcal{X}\left(\tau_{i+1}-\tau\right) B v(\tau)\right)_{2} \mathrm{~d} \tau \\
& \leq \int_{\tau_{i}}^{\tau_{i+1}}\left(\tilde{s}_{i}, B u^{h}(\tau)\right)_{2} \mathrm{~d} \tau \\
& -\int_{\tau_{i}}^{\tau_{i+1}}\left(\tilde{s}_{i}, B v(\tau)\right)_{2} \mathrm{~d} \tau+K^{(0)} \mu\left(\delta_{i}\right) \delta_{i},
\end{aligned}
$$

Here, $\delta_{i}=\delta_{i}(h)$. In what follows, $K^{(j)}=$ const $>0$, $j=0,1,2$. Hence we have

$$
\begin{aligned}
\lambda_{i} \leq & 2 \exp \left(-2 \omega \tau_{i+1}\right) \\
& \times\left(\tilde{s}_{i}, \int_{\tau_{i}}^{\tau_{i+1}} B\left\{u^{h}(\tau)-v(\tau)\right\} \mathrm{d} \tau\right)_{2} \\
& +K^{(1)}\left(\nu_{i}^{h}+\mu\left(\delta_{i}\right)\right) \delta_{i} .
\end{aligned}
$$

From (19), using (17), we obtain

$$
\lambda_{i} \leq K^{(1)}\left(\nu_{i}^{h}+\mu\left(\delta_{i}\right)\right) \delta_{i} .
$$

Thus, instead of the inequality 15 , we have the inequality (20). Consequently, in lieu of the inequality (16), we obtain

$$
\begin{aligned}
\varepsilon_{h}\left(\tau_{i}\right) \leq & \varepsilon_{h}(0)+K^{(2)} \sum_{j=0}^{\infty} \delta_{j}(h)\left\{\nu_{j}^{h}+\delta_{j}(h)\right. \\
& \left.+\mu\left(\delta_{j}(h)\right)\right\}, \quad j=1,2, \ldots .
\end{aligned}
$$

Hence, we derive the assertion of the theorem. The proof is complete.
Under some additional conditions on the semigroup $\mathcal{X}(t)$, the calculation procedure for the control $u^{h}(\tau)$, $\tau \in\left[\tau_{i}, \tau_{i+1}\right)$, can be simplified. Let us proceed with the description of these conditions.

Condition 5. The semigroup $\mathcal{X}(t)$ possesses the following property. For any bounded set $X_{*} \subset X$, there exist numbers $\delta_{*} \in(0,1)$ and $k_{0}=k_{0}\left(X_{*}\right) \in(0,+\infty)$ such that

$$
\left|\left(\mathcal{X}(\delta) x, \mathcal{X}\left(\delta_{1}\right) B v\right)_{2}-(x, B v)_{2}\right| \leq k_{0} \gamma(\delta),
$$

uniformly with respect to all $x \in X_{*}, \delta \in\left(0, \delta_{*}\right)$, $\delta_{1} \in[0, \delta]$, and $v \in P$, where $\gamma(\cdot):\left[0, \delta_{*}\right) \rightarrow R$ is a nonnegative function continuous at zero and $\gamma(0)=0$.

Theorem 3. Let Conditions 4 and 5 be satisfied and the assumptions of Theorem 1 hold as well. Let also the function $u^{h}(\cdot)$ be determined by the rule

$$
u^{h}(t)=u_{i}^{h} \quad \text { for a.a. } t \in\left[\tau_{i}, \tau_{i+1}\right),
$$

where

$$
\left(s_{i}, B u_{i}^{h}\right)_{2} \leq \inf \left\{\left(s_{i}, B u\right)_{2}: u \in P\right\}+d \nu_{i}^{h} .
$$

Then the assertion of Theorem 2 is valid if

$$
\varphi_{3}(h)=\sum_{i=0}^{\infty} \delta_{i}(h) \gamma\left(\delta_{i}(h)\right) \rightarrow 0 \quad \text { as } h \rightarrow+0 .
$$

Proof. Indeed, by Condition 5, we get

$$
\begin{aligned}
& \int_{\tau_{i}}^{\tau_{i+1}}\left(\tilde{s}_{i}, \mathcal{X}\left(\tau_{i+1}-\tau\right) B u^{h}(\tau)\right)_{2} \mathrm{~d} \tau \\
& -\int_{\tau_{i}}^{\tau_{i+1}}\left(\tilde{s}_{i}, \mathcal{X}\left(\tau_{i+1}-\tau\right) B v(\tau)\right)_{2} \mathrm{~d} \tau \\
& \leq \int_{\tau_{i}}^{\tau_{i+1}}\left(\xi_{i}^{h}-\psi_{i}^{h}, B u^{h}(\tau)\right)_{2} \mathrm{~d} \tau \\
& -\int_{\tau_{i}}^{\tau_{i+1}}\left(\xi_{i}^{h}-\psi_{i}^{h}, B v(\tau)\right)_{2} \mathrm{~d} \tau+K_{0} \gamma\left(\delta_{i}\right) \delta_{i} .
\end{aligned}
$$

Here $\delta_{i}=\delta_{i}(h)$ and $K_{0}=$ const $>0$. Hence, instead of the inequality (19), we have

$$
\begin{aligned}
\lambda_{i} \leq & 2 \exp \left(-2 \omega \tau_{i+1}\right) \\
& \times\left(\tilde{s}_{i}, \int_{\tau_{i}}^{\tau_{i+1}} B\left\{u^{h}(\tau)-v(\tau)\right\} \mathrm{d} \tau\right)_{2} \\
& +K_{1}\left(\nu_{i}^{h}+\gamma\left(\delta_{i}\right)\right) \delta_{i} .
\end{aligned}
$$


From (22) it follows that

$$
\begin{aligned}
& \exp \left(-2 \omega \tau_{i+1}\right)\left(s_{i}, B u_{i}^{h}\right)_{2} \\
& \leq \inf \left\{\exp \left(-2 \omega \tau_{i+1}\right)\left(s_{i}, B u\right)_{2}: u \in P\right\} \\
& \quad+K_{2} \nu_{i}^{h} .
\end{aligned}
$$

Therefore, on account of (23) and (24), we get

$$
\begin{aligned}
\varepsilon_{h}\left(\tau_{i}\right) \leq & \varepsilon_{h}(0)+K_{2} \sum_{j=0}^{\infty} \delta_{j}(h)\left\{\nu_{j}^{h}+\delta_{j}(h)\right. \\
& +\gamma\left(\delta_{i}(h)\right\}, \quad i=1,2, \ldots
\end{aligned}
$$

Hence, we obtain the assertion of the theorem. This completes the proof.

\section{Example}

Consider the differential-functional equation

$$
\begin{aligned}
\dot{y}(t)= & \sum_{k=0}^{l} A_{k} y\left(t-\nu_{i}\right)+\int_{-\nu_{l}}^{0} A_{*}(s) y(t+s) \mathrm{d} s \\
& +B_{0} u(t), \quad t \in T
\end{aligned}
$$

with the initial condition $y\left(t_{0}\right)=\varphi^{0}, y\left(t_{0}+s\right)=\varphi^{1}(s)$ for a. a. $s \in\left[-\nu_{l}, 0\right]$.

Here $y(t) \in \mathbb{R}^{n}, u(t) \in \mathbb{R}^{m}, \varphi^{0} \in \mathbb{R}^{n}, \varphi^{1}(\cdot) \in$ $L_{2}\left(\left[-\nu_{l}, 0\right] ; \mathbb{R}^{n}\right), 0=\nu_{0}<\nu_{1}<\ldots<\nu_{l}<+\infty$, $y_{t}(\cdot): s \rightarrow y(t+s),-\nu_{l} \leq s \leq 0, A_{k}, k \in[0: l]$, and $B_{0}$ are constant $n \times n$ and $n \times m$ matrices, respectively. The elements of the matrix function $s \rightarrow A_{*}(s), s \in\left[-\nu_{l}, 0\right]$, belong to the space $L_{\infty}\left(\left[-\nu_{l}, 0\right] ; \mathbb{R}\right), U=\mathbb{R}^{m}$.

Denote by $X=\mathbb{R}^{n} \times L_{2}\left(\left[-\nu_{l}, 0\right] ; R^{n}\right)$ the Hilbert space of pairs $x=\left\{x^{0}, x^{1}(s)\right\}$ with the inner product

$$
(x, y)_{X}=\left(x^{0}, y^{0}\right) \mathbb{R}^{n}+\int_{-\nu_{l}}^{0}\left(x^{1}(s), y^{1}(s)\right)_{\mathbb{R}^{n}} \mathrm{~d} s
$$

and the norm $|\cdot|_{X}$ induced by this inner product.

Equation 25) (see Banks and Kappel, 1979; Bernier and Manitius, 1978) generates the $C_{0}$-semigroup of bounded linear operators $\mathcal{X}(t), t \geq 0$, defined as follows. Let $s_{0}(\cdot)$ be the unique solution on $T$ of the matrix equation

$$
\begin{aligned}
\frac{\mathrm{d} s_{0}(t)}{\mathrm{d} t}= & A_{0} s_{0}(t)+\sum_{i=1}^{l} A_{i} s_{0}\left(t+\nu_{i}\right) \\
& +\int_{-\nu_{l}}^{0} A_{*}(s) s_{0}(t+s) \mathrm{d} s \quad \text { for a. a. } t \in T
\end{aligned}
$$

with the initial condition $s_{0}(t)=E$ (the $n \times n$ identity matrix) for $t \leq 0$. Assume that $B_{*}: L_{2}\left(\left[-\nu_{l}, 0\right] ; \mathbb{R}^{n}\right) \rightarrow$
$L_{2}\left(\left[-\nu_{l}, 0\right] ; \mathbb{R}^{n}\right)$ is an operator of the form

$$
\begin{aligned}
\left(B_{*} \varphi\right)(\tau)= & \sum_{i=1}^{l} A_{i} \chi_{\left[-\nu_{i}, 0\right]}(\tau) \varphi\left(-\nu_{i}-\nu\right) \\
& +\int_{-\nu_{l}}^{0} A_{*}(\xi) \varphi(\xi-\tau) \mathrm{d} \xi
\end{aligned}
$$

for almost all $\tau \in\left[-\nu_{l}, 0\right], \chi_{[a, b]}(\cdot)$ is the characteristic function of the interval $[a, b]$, and the operator $F: X \rightarrow$ $X$ is defined by the rule

$$
(F \varphi)^{0}=\varphi^{0},(F \varphi)^{1}=B_{1} \varphi^{1}\left(\varphi=\left\{\varphi^{0}, \varphi^{1}(s)\right\} \in X\right) .
$$

Then (Bernier and Manitius, 1978, p. 903)

$$
\mathcal{X}(t) \varphi=G_{t} F \varphi+S(t) \varphi
$$

where $G_{t}: X \rightarrow X, S(t): X \rightarrow X$,

$$
\begin{gathered}
(S(t) \varphi)^{0}=0, \quad(S(t) \varphi)^{1}(\tau)=\varphi(t+\tau) \chi_{\left[-\nu_{l},-t\right)}(\tau), \\
\left(G_{t} \varphi\right)^{0}=\left(G_{t} \varphi\right)^{1}(0), \\
\left(G_{t} \varphi\right)^{1}(\tau)=s_{0}(t+\tau) \varphi^{0} \\
+\int_{-\nu_{l}}^{0} s_{0}(t+\tau+\xi) \varphi^{1}(\xi) \mathrm{d} \xi \\
\tau \in\left[-\nu_{l}, 0\right] .
\end{gathered}
$$

In this case, the operator $A$ is given by the relations (Bernier and Manitius, 1978, Assertion 2.1)

$$
\begin{aligned}
D(A)= & \left\{\varphi=\left\{\varphi^{0}, \varphi^{1}(s)\right\} \in X:\right. \\
& \left.\varphi^{1}(\cdot) \in W^{1,2}\left(\left[-\nu_{l}, 0\right] ; \mathbb{R}^{n}\right), \varphi^{1}(0)=\varphi^{0}\right\}, \\
A(\varphi)= & \left\{L\left(\varphi^{1}\right), \dot{\varphi}^{1}\right\}, \quad \varphi=\left\{\varphi^{0}, \varphi^{1}(\cdot)\right\} \in D(A) .
\end{aligned}
$$

Here $W^{1,2}\left([a, b] ; \mathbb{R}^{n}\right)$ means the space of $\mathbb{R}^{n}$-valued absolutely continuous functions with derivatives from the space $L_{2}\left([a, b] ; \mathbb{R}^{n}\right)$. Denote by $\mathcal{O}$ the zero element of the space $L_{2}\left(\left[-\nu_{l}, 0\right]\right.$. Let the linear continuous operator $B: U \rightarrow X$ have the form

$$
\begin{aligned}
B u=\left\{B_{0} u, \mathcal{O}\right\} \in & X \\
& \left(u \in U, \mathcal{O} \in L_{2}\left(\left[-\nu_{l}, 0\right] ; \mathbb{R}^{n}\right)\right) .
\end{aligned}
$$

Let $y\left(\cdot ; 0,\left\{\varphi^{0}, \varphi^{1}(\cdot)\right\}, u(\cdot)\right)$ be the unique solution of Eqn. 25 in the sense of Caratheodory, and let $x\left(\cdot ; 0, x_{0}, u(\cdot)\right)$ be the weak solution of the abstract differential equation (1), where $x_{0}=\left\{\varphi^{0}, \varphi^{1}(\cdot)\right\} \in X$. Then, for all $x_{0}=\left\{\varphi^{0}, \varphi^{1}(\cdot)\right\} \in X, u(\cdot) \in L_{\infty}(T ; U)$ and $t \in T$, we have

$$
\begin{aligned}
x\left(t ; 0, x_{0}, u(\cdot)\right)= & \left\{y\left(t ; 0,\left\{\varphi^{0}, \varphi^{1}(\cdot)\right\}, u(\cdot)\right),\right. \\
& \left.y_{t}\left(\cdot ; 0,\left\{\varphi^{0}, \varphi^{1}(\cdot)\right\}, u(\cdot)\right)\right\}
\end{aligned}
$$


is valid.

In the space $X$, we define the norm $|\cdot|_{2}$ as follows:

$$
\begin{gathered}
\left|\left\{\varphi^{0}, \varphi^{1}(\cdot)\right\}\right|_{2}=\left(\left|\varphi^{0}\right|_{\mathbb{R}^{n}}^{2}+\int_{-\nu_{l}}^{0}\left|\varphi^{1}(\tau)\right|_{\mathbb{R}^{n}}^{2} g(\tau) \mathrm{d} \tau\right)^{1 / 2}, \\
\left\{\varphi^{0}, \varphi^{1}(\cdot)\right\} \in X,
\end{gathered}
$$

where $g(\tau)=j$ for $j \in\left(-\nu_{l-j+1},-\nu_{l-j}\right), j \in[1$ : $l]$. The inner product corresponding to this norm is of the form

$$
\begin{aligned}
& \left(\left\{\varphi^{0}, \varphi^{1}(\cdot)\right\},\left\{\bar{\varphi}^{0}, \bar{\varphi}^{1}(\cdot)\right\}\right)_{2} \\
& \quad=\left(\varphi^{0}, \bar{\varphi}^{0}\right)_{\mathbb{R}^{n}}+\int_{-\nu_{l}}^{0} g(\tau)\left(\varphi^{1}(\tau), \bar{\varphi}^{1}(\tau)\right)_{\mathbb{R}^{n}} \mathrm{~d} \tau .
\end{aligned}
$$

In this case (Bernier and Manitius, 1978, Lemma 2.3,), the semigroup $\mathcal{X}(t), t \in T$, of form (26) is $\omega$-dissipative. In addition,

$$
\omega=\frac{l+1}{2}+\left|A_{0}\right|_{*}+\frac{1}{2} \sum_{i=1}^{l}\left|A_{i}\right|_{*}^{2}+\frac{1}{2} \int_{-\nu_{l}}^{0}\left|A_{*}(\tau)\right|_{*}^{2} \mathrm{~d} \tau .
$$

Here, the symbol $|\cdot|_{*}$ denotes the Euclidean norm of a matrix. It can be easily verified that (Maksimov, 2002, see Lemma 2.1.8,), the semigroup $\mathcal{X}(t), t \in T$, of the form (26) satisfies Condition 5 for $\gamma(\delta)=\delta^{1 / 2}$.

In addition, in the inequality (22) we have

$$
\left(s_{i}, B u\right)_{2}=\left(y\left(\tau_{i}\right)-\xi\left(\tau_{i}\right)\right)^{\prime} B_{0} u,
$$

where

$$
s_{i}=\left\{y\left(\tau_{i}\right)-\xi\left(\tau_{i}\right), \mathcal{O}\right\} \in X, \quad \mathcal{O} \in L_{2}\left(\left[-\nu_{l}, 0\right] ; \mathbb{R}^{n}\right) .
$$

Here, $\xi\left(\tau_{i}\right) \in \mathbb{R}^{n}$ is the result of measurement of the state $y\left(\tau_{i}\right)=y\left(\tau_{i} ;\left\{\varphi^{0}, \varphi^{1}(\cdot)\right\}, u(\cdot)\right),\left|y\left(\tau_{i}\right)-\xi\left(\tau_{i}\right)\right|_{\mathbb{R}^{n}} \leq \nu_{i}^{h}$ and the prime stands for transposition.

\section{Numerical example}

In this section we present a numerical example. Consider the equation

$$
\begin{gathered}
\frac{\partial}{\partial t} x(\nu, t)-\Delta_{L} x(\nu, t)=u(\nu, t) \quad \text { in } Q=\Omega \times T \\
x(0, t)=0, \quad x(1, t)=0 \quad \text { in } T \\
x(\nu, 0)=x_{0}(\nu) \quad \text { in } \Omega
\end{gathered}
$$

where $\Omega=(0,1), T=[0, \vartheta], \Delta_{L} x=\partial^{2} x(\nu) / \partial \nu^{2}, \vartheta=$ 10. As is well known, this equation can be written in the form (1). It is assumed that we have the second (Eqn. (4))

$$
\frac{\partial}{\partial t} y(\nu, t)-\Delta_{L} y(\nu, t)=v(\nu, t) \quad \text { in } Q
$$

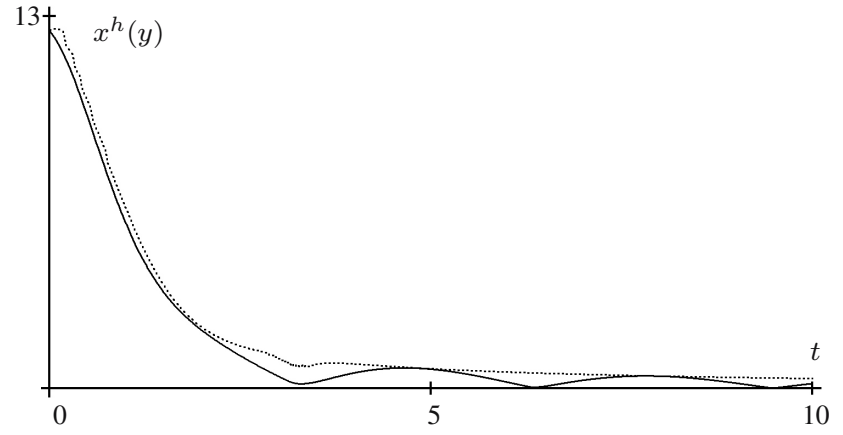

Fig. 1. $h=0.5$.

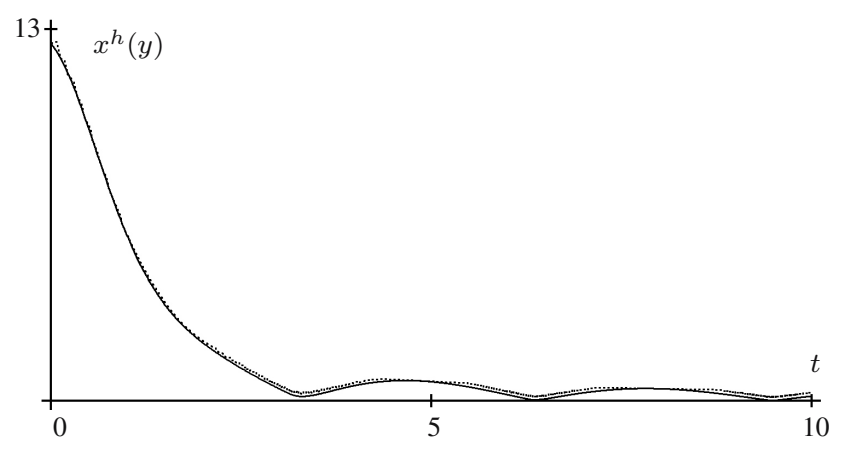

Fig. 2. $h=0.1$.

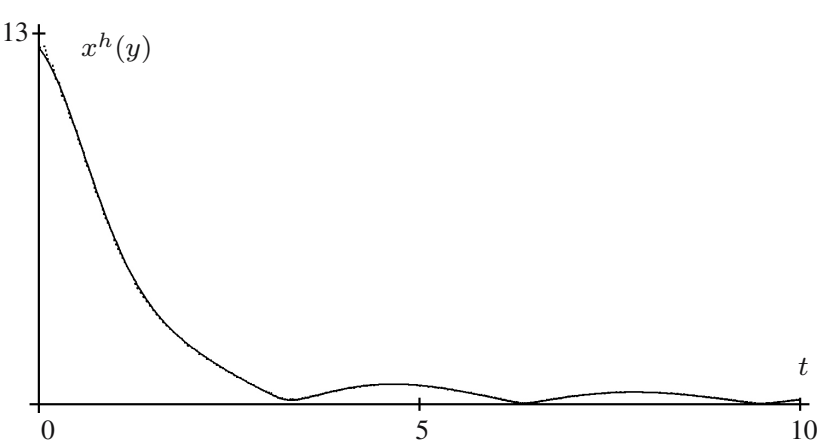

Fig. 3. $h=0.01$.

$$
\begin{gathered}
y(0, t)=y(1, t)=0 \quad \text { in } T, \\
y(\nu, 0)=y(\nu) \quad \text { in } \Omega
\end{gathered}
$$

influenced by the action of an unknown disturbance $v=$ $v(\nu, t) \in P$. The problem is to design a control

$$
\begin{aligned}
u(t)=u^{h}(t)=\mathcal{U}\left(\tau_{i}, \xi_{i}^{h}, \psi_{i}^{h}\right) \subset P & \\
& \text { for } t \in\left[\tau_{i}, \tau_{i+1}\right)
\end{aligned}
$$

yielding small values of

$$
W\left(x^{h}\left(\cdot ; 0, x_{0}, u^{h}(\cdot)\right), y(\cdot ; 0, y, v(\cdot)) \mid \vartheta\right) .
$$

Here $x^{h}(\cdot)=x^{h}\left(\cdot ; 0, x_{0}, u^{h}(\cdot)\right)$ is the solution of Eqn. (28) corresponding to a control $u(\cdot)=u^{h}(\cdot)$. 
In the experiment, we set

$$
\begin{aligned}
P & =\{\pi(\nu) u:|u| \leq 1\}, \\
\pi(\nu) & =0.5 \nu(\nu-1), \quad \nu \in[0,1] .
\end{aligned}
$$

The control on the right-hand part of Eqn. (28) is calculated by the formulas (30), (17), (18) under the assumption that

$$
u^{h}(t)= \begin{cases}1 & \text { if }\left(\xi_{i}^{h}-\psi_{i}^{h}, \pi\right)_{L_{2}(\Omega)} \leq 0 \\ -1 & \text { if }\left(\xi_{i}^{h}-\psi_{i}^{h}, \pi\right)_{L_{2}(\Omega)}>0\end{cases}
$$

Equations (28) and (20) are solved by the set method with the step $\Delta \omega$ in the domain $\Omega$. In Figs. 1 and 2 the results of computer modeling are presented for the following case:

$$
\begin{gathered}
\Delta \omega=\frac{1}{10}, \quad \delta_{i}(h)=\frac{h}{(i+1)^{\mu}}, \quad \mu=\frac{3}{4}, \\
x_{0}(\nu)=25 j \Delta \omega(1-j \Delta \omega), \quad y_{0}(\nu)=x_{0}(\nu)+h, \\
j=0, \ldots, n ; \quad n=10, \\
v(\nu, t)=0.5 \nu(\nu-1) v(t), \quad v(t)=|\sin t| .
\end{gathered}
$$

During the experiment, we have assumed

$$
\begin{aligned}
\xi_{i}^{h}\left(\nu_{j}\right) & =x^{h}\left(\nu_{j}, \tau_{i}\right)+h, \\
\psi_{i}^{h}\left(\nu_{j}\right) & =y\left(\nu_{j}, \tau_{i}\right)+h,
\end{aligned}
$$

where $\nu_{j}=j \Delta \omega, j=0, \ldots, 1 / \Delta \omega$. Figure 1 corresponds to the case when $h=0.5$, Fig. 2 to the case when $h=0.1$, and Fig. 3 to the case when $h=0.01$. In these figures the solid line represents the function $x^{h}(t, \nu)=x^{h}\left(t, \nu ; 0, x_{0}, u^{h}(\cdot)\right)$ and the dotted line represents $y(t, \nu)=y\left(t, \nu ; 0, y_{0}, v(\cdot)\right)$ for $\nu=0.5$.

\section{Acknowledgment}

The work was supported in part by the Russian Foundation for Basic Research under the grant 17-01-00042a.

\section{References}

Banks, H.T. and Kappel, F. (1979). Spline approximation for functional-differential equations, Journal of Differential Equations 34(3): 406-522.

Bernier, C. and Manitius, A. (1978). On semigroups in $\mathbb{R}^{n} \times L^{p}$ corresponding to differential equations with delays, Canadian Journal of Mathematics 30(5): 897-914.

Blizorukova, M., Kappel, F. and Maksimov, V. (2001). A problem of robust control of a system with time delay, International Journal of Applied Mathematics and Computer Science 11(4): 821-834.

Grimble, J.M., Johnson, M.A. (1988). Optimal Control and Stochastic Estimation: Theory and Applications, John Wiley \& Sons, Chichester.
Kapustyan, V. and Maksimov, V. (2014). On attaining the prescribed quality of a controlled fourth order system, International Journal of Applied Mathematics and Computer Science 24(1): 75-85, DOI: 10.2478/amcs-2014-0006.

Krasovskii, N.N. and Subbotin, A.I. (1988). Game-Theoretical Control Problems, Springer Verlag, New York, NY/Berlin.

Kryazhimskiy, A.V. and Maksimov, V.I. (2011). Resource-saving tracking problem with infinite time horizon, Differential Equations 47(7): 1004-1013.

Maksimov, V.I. (2011). The tracking of the trajectory of a dynamical system, Journal of Applied Mathematics and Mechanics 75(6): 667-674.

Maksimov, V.I. (2002). Dynamic Inverse Problems of Distributed Systems, VSP, Utrecht/Boston, MA.

Maksimov, V.I. (2012). On tracking solutions of parabolic equations, Russian Mathematic 56(1): 35-42.

Maksimov, V.I. (2013). Regularized extremal shift in problems of stable control, in D. Hömberg and F. Tröltzsch (Eds.), IFIP Advances in Information and Communication Technology, Vol. 391, Springer, Berlin, pp. 112-121.

Maksimov, V.I. (2014). Algorithm for shadowing the solution of a parabolic equation on an infinite time interval, Differential Equations 50(3): 362-371.

Osipov, Yu.S. (2009). Selected Works, Moscow State University, Moscow.

Pandolfi, L. and Priola, E. (2005). Tracking control of parabolic systems, Proceedings of the 21st IFIP TC7 Conference on System Modeling and Optimization, Sophia Antipolis, France, pp. 135-146.

Prodan, I., Olaru, S., Stoica, C., and Niculescu, S.-I. (2013). Predictive control for trajectory tracking and decentralized navigation of multi-agent formations, International Journal of Applied Mathematics and Computer Science 23(1): 91-102, DOI: 10.2478/amcs-2013-0008.

Sontag, E.D. (1990). Mathematical Control Theory, Springer Verlag, Berlin.

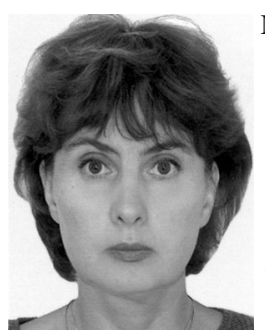

Marina Blizorukova received her $\mathrm{PhD}$ degree in differential equations from the Institute of Mathematics and Mechanics (Yekaterinburg, Russia) in 2001. Currently, she is a staff scientific researcher of the Institute of Mathematics and Mechanics, Ural Branch of the Russian Academy of Sciences, and an associate professor in the Department of Economics and Management, Ural Federal University, Russia. She is an author or a co-author of several papers in the field of inverse problems, mathematical modeling, optimal control and their applications in various areas. 


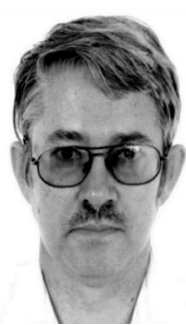

Vyacheslav Maksimov graduated in mathematics and mechanics from the Ural State University, Yekaterinburg, Russia, in 1972. He received the $\mathrm{PhD}$ and $\mathrm{DSc}$ degrees from the Institute of Mathematics and Mechanics, Ural Branch of the Russian Academy of Sciences, in 1978 and 1992, respectively. Since 1972, he has been with the Institute of Mathematics and Mechanics, Yekaterinburg, Russia. Since 1994, he has been the head of a department at the same institute and a professor at the Chair of Controlled Systems Modeling of the Ural Federal University, Yekaterinburg. He is the author of more than 100 technical publications, including three monographs. His research interests are primarily focused on control theory, distributed parameter systems, and mathematical modeling. Dr. Maksimov is a member of the American Mathematical Society and of the IFIP WG7.2. He has been on editorial boards of several journals.

Received: 30 November 2016

Revised: 13 March 2017

Accepted: 4 May 2017 